\title{
Phase diagram and influence of defects in the double perovskites
}

\author{
J. L. Alonso, ${ }^{1,2}$ L. A. Fernández, ${ }^{3,2}$ F. Guinea, ${ }^{4,2}$ F. Lesmes, ${ }^{5}$ and V. Martín-Mayor ${ }^{3,2}$ \\ ${ }^{1}$ Departamento de Física Teórica, Facultad de Ciencias, Universidad de Zaragoza, 50009 Zaragoza, Spain \\ ${ }^{2}$ Instituto de Biocomputación y Física de Sistemas Complejos (BIFI), Universidad de Zaragoza, 50009 Zaragoza, Spain \\ ${ }^{3}$ Departamento de Física Teórica, Facultad de Ciencias Físicas, Universidad Complutense de Madrid, 28040 Madrid, Spain \\ ${ }^{4}$ Instituto de Ciencia de Materiales (CSIC), Cantoblanco, 28049 Madrid, Spain \\ ${ }^{5}$ Centro de Astrobiología, INTA-CSIC, Carretera de Ajalvir km 4, 28850 Torrejón de Ardoz, Madrid, Spain
}

(Received 27 January 2003; published 19 June 2003)

\begin{abstract}
The phase diagram of the double perovskites of the type $\mathrm{Sr}_{2-x} \mathrm{La}_{x} \mathrm{FeMoO}_{6}$ is analyzed, with and without disorder due to antisites. In addition to an homogeneous half metallic ferrimagnetic phase in the absence of doping and disorder, we find antiferromagnetic phases at large dopings, and other ferrimagnetic phases with lower saturation magnetization, in the presence of disorder.
\end{abstract}

DOI: 10.1103/PhysRevB.67.214423

PACS number(s): 75.47.Gk, 75.10.-b

\section{INTRODUCTION}

The double perovskite $\mathrm{Sr}_{2} \mathrm{FeMoO}_{6}$ and related materials ${ }^{1}$ are good candidates for magnetic devices, as they combine a high Curie temperature and a fully polarized (half metallic) conduction band. ${ }^{2}$ At present, these materials are being extensively studied. ${ }^{3-15}$

The magnetism of these compounds arises from the $\mathrm{Fe}^{3+}$, $S=5 / 2$ core spin, while the charge state of the Mo ion is 5 + . Spatially, the Mo and Fe ions occupy two interleaving fcc lattices (sodium chloride structure). The conduction band contains one electron per unit cell, which tends to be antiparallel to the Fe spin. Experiments suggest that, in many samples, the saturation magnetization is less than the expected $4 \mu_{B}$ per formula unit. This effect is usually ascribed to the presence of antisite defects, $3,5,8-11,13,14,16,17$ where, due to the similarity of their atomic radii, Mo ions are randomly placed on the Fe sublattice and conversely. Notice that when a Fe ion is misplaced, with high probability it will have a Fe ion among its first neighbors, enhancing direct antiferromagnetic (AFM) superexchange with respect to the ideal structure. The strength of this coupling can be inferred from the compound $\mathrm{LaFeO}_{3}$, which has the same structure, but where the Mo ions have been substituted by $\mathrm{Fe}^{3+} \cdot \mathrm{LaFeO}_{3}$ is known to be $\mathrm{AFM},{ }^{18}$ with a Néel temperature of $T_{N}$ $=720 \mathrm{~K}$.

The $\mathrm{Sr}$ ions in $\mathrm{Sr}_{2} \mathrm{FeMoO}_{6}$ can be substituted for trivalent cations, like La, leading to $\mathrm{Sr}_{2-x} \mathrm{La}_{x} \mathrm{FeMoO}_{6} \cdot{ }^{4,8,13}$ These compounds have $1+x$ electrons per formula unit in the conduction band. These doped materials tend to have a higher Curie temperature. Notice that one can also consider the substitution with a monovalent ion (i.e., $\mathrm{Sr} \rightarrow \mathrm{K}, \mathrm{Sr}_{2-x} \mathrm{~K}_{x} \mathrm{FeMoO}_{6}$ ), which takes one electron from the conduction band, leaving $1-x$ electrons per formula unit. Hence in this paper negative $x$ will actually refer to substitution with a monovalent ion.

\section{THE MODEL}

\section{A. Clean system}

Band structure calculations have shown that the conduction band can be described in terms of hybridized $\boldsymbol{t}_{2 g}$ orbitals at the Mo and Fe sites. ${ }^{2,19-21}$ If one considers the $\boldsymbol{t}_{2 g}$ orbitals of both spin orientations at the Fe sites, the model leads to a highly correlated system, where an on site Hund's coupling and a Hubbard repulsive term have to be added. ${ }^{22-24}$ In the following, we will consider the magnetic phase diagram only, and neglect the possible existence of a metal-insulator transition when the ratio between the bandwidth and the Coulomb term is sufficiently small. ${ }^{23,24}$ We consider that the conduction band is built up of the three $\boldsymbol{t}_{2 g}$ orbitals at the Fe sites with spins oriented antiparallely to the Fe moment, and the six $\boldsymbol{t}_{2 g}$ orbitals at the Mo sites (see below).

We denote the destruction operator on $x y$ orbitals with spin + or - at lattice site $\boldsymbol{r}$ as $F_{x y ; \uparrow, \downarrow ; r}, M_{x y ; \uparrow, \downarrow ; r}(F$ for Fe and $M$ for Mo), and so on. The total spin and number operators on a given Fe site are

$$
\begin{gathered}
\overrightarrow{\mathcal{S}}_{\boldsymbol{r}}=\sum_{\alpha, \beta=\uparrow, \downarrow}\left(F_{x y ; \alpha ; \boldsymbol{r}}^{\dagger}+F_{x z ; \alpha ; \boldsymbol{r}}^{\dagger}+F_{y z ; \alpha ; \boldsymbol{r}}^{\dagger}\right) \\
\times \vec{\sigma}_{\alpha, \beta}\left(F_{x y ; \beta ; \boldsymbol{r}}+F_{x z ; \beta ; \boldsymbol{r}}+F_{y z ; \beta ; \boldsymbol{r}}\right), \\
\mathcal{N}_{\boldsymbol{r}}^{\mathrm{Fe}}=\sum_{\alpha=\uparrow, \downarrow}\left(F_{x y ; \alpha ; \boldsymbol{r}}^{\dagger} F_{x y ; \alpha ; \boldsymbol{r}}+F_{x z ; \alpha ; \boldsymbol{r}}^{\dagger} F_{x z ; \alpha ; \boldsymbol{r}}+F_{y z ; \alpha ; \boldsymbol{r}}^{\dagger} F_{y z ; \alpha ; \boldsymbol{r}}\right) .
\end{gathered}
$$

Analogous definitions hold for the Mo atoms. Given the large spin value $(S=5 / 2)$ of the localized Fe core spins, we treat them as classical variables, with polar coordinates

$$
\vec{\phi}=(\sin \theta \cos \varphi, \sin \theta \sin \varphi, \cos \theta) .
$$

Physically this implies an adiabatic approximation, in the sense that charge carriers are assumed to be in thermal equilibrium with the instantaneous classical spin configuration.

As mentioned above, we only consider the Fe orbitals with spin antiparallel to $\vec{\phi}$, which amounts to assume that the Hund's coupling at the Fe ions is much larger than the other interactions. Thus, we define up and down orbitals, $f_{\uparrow}$ and $f_{\downarrow}$, with respect to the local $5 / 2$ spin

$$
F_{\uparrow}=\cos \frac{\theta}{2} f_{\uparrow}+\sin \frac{\theta}{2} f_{\downarrow},
$$




$$
F_{\downarrow}=\sin \frac{\theta}{2} e^{i \varphi} f_{\uparrow}-\cos \frac{\theta}{2} \mathrm{e}^{\mathrm{i} \varphi} f_{\downarrow},
$$

where the spin index of the $f$ operators is referred to the local direction of the Fe moment. In the following, we neglect all terms including the $f_{\uparrow}$ operators, since we treat the local Hund coupling as the dominant interaction (note that the $\mathbf{t}$ orbital with spin up at the Fe site is included in the Fe local moment). For the sake of brevity, we set $f_{\downarrow}=f$. Then, the Hamiltonian, in the absence of disorder and neglecting direct hopping terms between Mo orbitals (see below), can be written as

$$
\mathcal{H}=\mathcal{K}_{x y}+\mathcal{K}_{y z}+\mathcal{K}_{x z}-\mu \sum_{\boldsymbol{r} \text { even }} \mathcal{N}_{\boldsymbol{r}}^{\mathrm{Fe}}-(\mu+\Delta) \sum_{\boldsymbol{r} \text { odd }} \mathcal{N}_{\boldsymbol{r}}^{\mathrm{Mo}}
$$

with

$$
\begin{aligned}
\mathcal{K}_{x y}= & t_{\mathrm{Mo}-\mathrm{Fe}} \sum_{\substack{r \in \mathrm{Fe} \text { lattice } \\
\hat{u}= \pm \hat{e}_{x}, \pm \hat{e}_{y}}}\left[\left(\sin \frac{\theta_{\boldsymbol{r}}}{2} f_{x y ; \boldsymbol{r}}^{\dagger} M_{x y ; \uparrow ; \boldsymbol{r}+\hat{u}}+\text { H.c. }\right)\right. \\
& -\left(e^{\left.\left.i \varphi_{r} \cos \frac{\theta_{\boldsymbol{r}}}{2} f_{x y ; r}^{\dagger} M_{x y ; \downarrow ; \boldsymbol{r}+\hat{u}}+\text { H.c. }\right)\right] .}\right.
\end{aligned}
$$

The effective hoppings are mediated by virtual jumps into orbitals at the oxygen sites lying between the Mo and Fe ions. The only allowed hoppings are from $\mathbf{t}_{x y}$ orbitals at $\mathrm{Fe}$ ions to $\mathbf{t}_{x y}$ orbitals at Mo ions, and within a given $x y$ plane. Analogous expressions are found for the kinetic energy on the $x z$ and $y z$ planes. Finally, we add a direct hopping between Mo orbitals located at Mo ions which are nearest neighbors in the Mo sublattice, as suggested by band structure calculations. ${ }^{19}$ These Mo-Mo hoppings are restricted, similar to the Mo-Fe ones, to pairs of orbitals with the same symmetry and within a given plane. This constraint does not apply to hoppings between orbitals located at more distant sites, which we do not consider here. The kinetic energy acquires new terms of the type

$$
\begin{aligned}
\mathcal{K}_{x y}^{\mathrm{Mo}}= & t_{\text {Mo-Mo }} \sum_{\substack{\alpha=\uparrow, \downarrow \\
r \in \text { Mo lattice }}}\left[\left(M_{x y ; \alpha ; r}^{\dagger} M_{x y ; \alpha ; r}+\hat{e}_{x}+\hat{e}_{y}+\text { H.c. }\right)\right. \\
& \left.+\left(M_{x y ; \alpha ; r}^{\dagger} M_{x y ; \alpha ; r+\hat{e}_{x}-\hat{e}_{y}}+\text { H.c. }\right)\right] .
\end{aligned}
$$

For computational purposes it will be extremelly convenient to notice that the total Hamiltonian can be decomposed into three sets of decoupled two dimensional Hamiltonians. ${ }^{22-24}$ Since the Hamiltonian is bilinear in the fermionic operators, and given the adiabatic assumption discussed after Eq. (3), it follows that the model can be studied using the techniques introduced in Refs. 26 and 27.

\section{B. Disordered systems}

The only source of disorder that we shall consider is the existence on antisite defects. ${ }^{3,5,8-11,13,14,17}$ That is, we shall consider the possibility that $\mathrm{Fe}$ atoms occupy nodes in the Mo lattice (and viceversa), in a random way. The substitution of Mo ions by $\mathrm{Fe}$ ions leads to the existence of pairs of $\mathrm{Fe}$ ions occupying nearest neighbor sites. This will modify the Hamiltonian in a twofold way.

(1) As said in the Introduction, the large Néel temperature ${ }^{18}$ of $\mathrm{LaFeO}_{3}(720 \mathrm{~K})$, suggests a strong antiferromagnetic superexchange coupling among the core spins $\vec{\phi}$. This antiferromagnetic coupling is usually disregarded in studies of the clean system, ${ }^{22}$ since superexchange is exponentially suppressed by the large distance between Fe ions in the perfect sodium chloridelike structure. However, the presence of antisite defects implies that $\mathrm{Fe}$ ions can get much closer than in the perfect structure, enhancing superexchange. Thus, we add to the Hamiltonian (6) an antiferromagnetic term

$$
J_{\mathrm{Fe}-\mathrm{Fe}} \sum^{\prime} \underset{\substack{\boldsymbol{r} \in \text { Mo lattice } \\ u} \hat{e}_{x}, \pm \hat{e}_{y}, \pm \hat{e}_{z}}{ } \vec{\phi}_{\boldsymbol{r}} \cdot \vec{\phi}_{\boldsymbol{r}+\hat{u}},
$$

where the sum is restricted to neighboring Fe pairs.

(2) The kinetic energy is also modified since now one can have Fe-Fe hopping through oxygen orbitals, for nearestneighbors Fe ions. This hopping is modulated by the spins just as in the double-exchange model (see, e.g., Ref. 27 for details):

$$
t_{\mathrm{Fe}-\mathrm{Fe}}\left[\cos \frac{\theta_{\boldsymbol{r}}}{2} \cos \frac{\theta_{\boldsymbol{r}+\hat{\mu}}}{2}+e^{i\left(\varphi_{\boldsymbol{r}}-\varphi_{\boldsymbol{r}+\hat{\mu}}\right)} \sin \frac{\theta_{\boldsymbol{r}}}{2} \sin \frac{\theta_{\boldsymbol{r}+\hat{\mu}}}{2}\right] .
$$

As discussed below, the magnetic moments of these pairs of neighboring $\mathrm{Fe}$ ions tend to be antiferromagnetically aligned, blocking the hopping of conduction electrons between them. Thus the value of the exchange constant $t_{\mathrm{Fe}-\mathrm{Fe}}$ is not a critical parameter. In the following we shall set $t_{\mathrm{Fe}-\mathrm{Fe}}=t_{\mathrm{Mo}-\mathrm{Fe}}$ for the sake of simplicity.

\section{Values of the parameters}

The model is defined by the parameters $t_{\mathrm{Mo}-\mathrm{Fe}}, t_{\mathrm{Mo}-\mathrm{Mo}}, \Delta, \mu$, and $J_{\mathrm{Fe}-\mathrm{Fe}}$. There are nine orbitals per unit cell, three at the Fe sites, and six at the Mo sites.

The occupancy of the conduction band depends on the value of the chemical potential, $\mu$, and it varies from one electron to two electrons per unit cell in $\mathrm{Sr}_{2-x} \mathrm{La}_{x} \mathrm{FeMoO}_{6}$, $0 \leqslant x \leqslant 1$. We neglect interactions of the electrons within this band. As discussed below, the number of electrons at the $\mathrm{Fe}$ sites is always less than one, making other couplings at the Fe sites irrelevant.

The cases to be considered in the following lead to some situations where the Mo ions have a finite probability of being doubly occupied, that is, in a $\mathrm{Mo}^{4+}$ state. We will not consider interactions between electrons at the Mo sites, however. Mo can exist in many oxidation states, implying that double occupancy is not strongly suppressed by Coulomb interactions. In addition, compounds of the type $R \mathrm{Mo}_{8} \mathrm{O}_{14}$ ( $R$ a rare earth) show no magnetic contribution from the Mo ions, which suggest weak magnetic interactions in Mo ions. ${ }^{25}$ We do not consider additional interactions at $\mathrm{O}$ sites. ${ }^{21}$

The leading interactions between different sites not considered here are probably superexchange couplings between $\mathrm{Fe}$ ions at the $\mathrm{Fe}$ sublattice ${ }^{20}$ (as discussed before, magnetic 
couplings between an Fe ion at a Mo site and its Fe neighbors are taken into account). It has been argued that these couplings can destabilize the ferrimagnetic state observed experimentally, although they may be weakened by lattice distortions. ${ }^{20}$ Our aim is not to obtain the phase diagram from first principles, but rather to analyze the influence of doping and defects on the possible magnetic phases, assuming the existence of a ferrimagnetic phase for $\mathrm{Sr}_{2} \mathrm{MoFeO}_{6}$. As discussed in Ref. 20, superexchange interactions between $\mathrm{Fe}$ ions in the $\mathrm{Fe}$ sublattice need to be canceled by other mechanisms in order to obtain a ferrimagnetic ground state for $\mathrm{Sr}_{2} \mathrm{MoFeO}_{6}$. Thus, the model that we describe here should suffice to understand the trends induced by doping and defects.

We will use $t_{\mathrm{Fe}-\mathrm{Mo}}$ as our unit of energy $\left(t_{\mathrm{Fe}-\mathrm{Mo}}\right.$ $\approx 0.35 \mathrm{eV}$ from band structure calculations). We take $t_{\mathrm{Mo}-\mathrm{Mo}} / t_{\mathrm{Fe}-\mathrm{Mo}}=0.25, \Delta=0$ and $J_{\mathrm{Fe}-\mathrm{Fe}} / t_{\mathrm{Fe}-\mathrm{Mo}}=0.1$. The value of $\Delta$ implies a relatively large hybridization of the $\mathrm{Fe}$ and Mo orbitals, which seems consistent with Hartree-Fock calculations. ${ }^{24} J_{\mathrm{Fe}-\mathrm{Fe}}$ is chosen so as to reproduce the Néel temperature of $\mathrm{LaFeO}_{3}$. We have not made a comprehensive study of the dependence of the results on the tight binding parameters, but the calculations made so far indicate that the qualitative features of the phase diagrams to be discussed below are not strongly dependent on the choice of parameters. In the absence of disorder, this model is basically equivalent to the one studied by Chattopadhyay and Millis ${ }^{22}$ in the context of dynamical mean field theory, although we shall use Variational Mean Field (see Ref. 26 for a comparison between the two methods). The main novelty is in our considering of the disorder effects: $:^{3,5,8-11,13,14,17}$ with probability $y$ we misplace an Fe ion onto the Mo sublattice (and conversely) without any spatial correlations ( $y$ is just the antisite density). It is clear that $y=0.5$ corresponds to full disorder on the location of the Fe and Mo ions, while $y$ $>0.5$ is equivalent to $1-y$ with the $\mathrm{Fe}$ and Mo sublattices interchanged. Vacancies can be equally considered, but explicit calculations showed that they have a much milder effect on the phase diagram.

\section{METHOD OF CALCULATION}

We use the method developed for double exchange systems in Ref. 26. We assume that the Fe core spins are classical. At a given temperature, we average over spin configurations obtained by assuming that there is a magnetic field acting on the spins. The magnitude of these fields are variational parameters, which are taken so as to minimize the free energy. Given a spin configuration, the electronic states are calculated exactly, and the electronic contribution to the free energy is obtained by integrating the density of states. As the $\mathrm{Fe}$ spins are distributed in a three-dimensional lattice, and the electrons lead to effective interactions with the cubic symmetry, we think that our mean field ansatz for the spin configurations is sufficient. This method is in excellent agreement with more precise Monte Carlo calculations for the double exchange model. ${ }^{27}$ We solve the Hamiltonian in lattices with up to $512 \times 512 \times 512$ sites (note that the calculation of the electronic wave functions requires only the di-

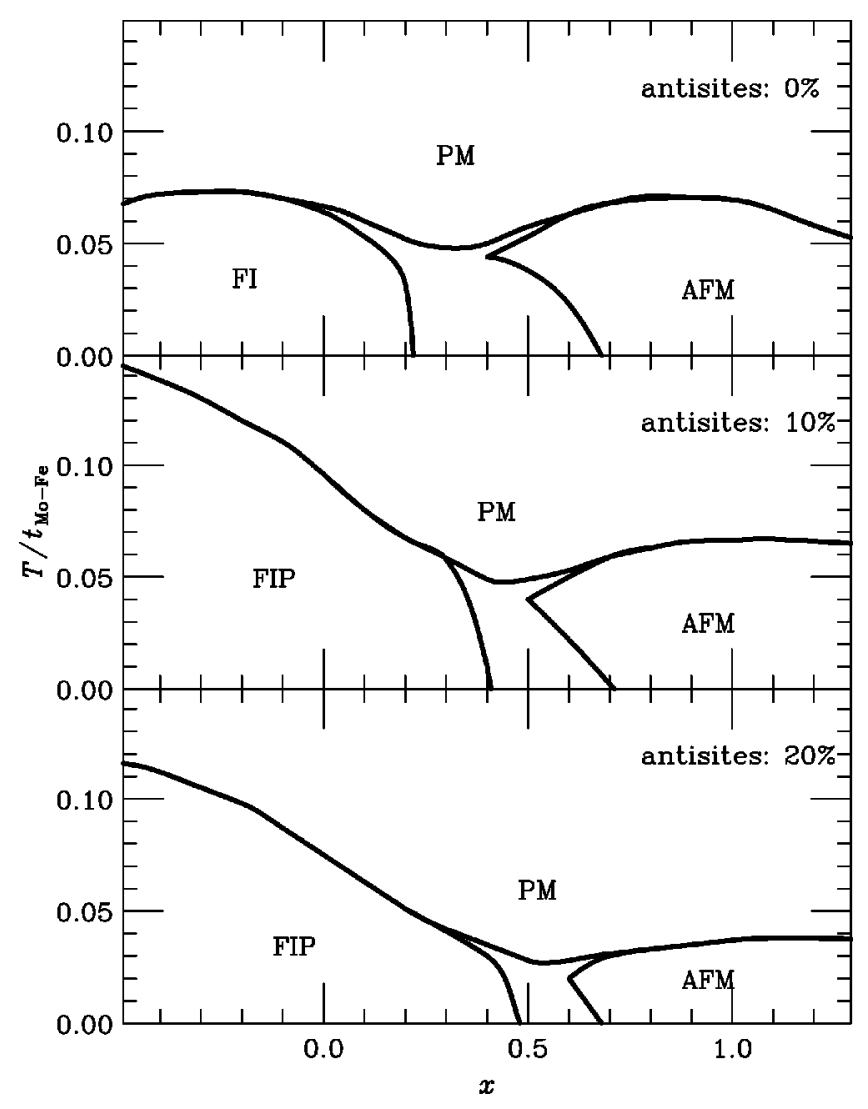

FIG. 1. Phase diagram of $\mathrm{Sr}_{2-x} \mathrm{La}_{x} \mathrm{FeMoO}_{6}$ as function of $x$ and temperature for different concentrations of antisite defects. Negative $x$ actually means $\mathrm{Sr}_{2-|x|} \mathrm{K}_{|x|} \mathrm{FeMoO}_{6}$. In both cases, the density of carriers in the conduction band is $1+x$. Phase-separation regions are found between the FI and AFM phases (upper panel), and between the FIP and AFM phases (middle and lower panel).

agonalization of the Hamiltonian in a $512 \times 512$ square). For these sizes, the disorder due to antisites is self-averaging.

The adequacy of our technique depends on the ansätze made for the possible spin configurations. We have considered four possible phases: (i) the paramagnetic (PM) phase, (ii) the ferrimagnetic $(\mathrm{FI})$ phase, where all $\mathrm{Fe}$ spins are parallel, and the spins of the electrons in the conduction band are antiparallel to the Fe spins, (iii) an AFM phase, where the Fe spins in neighboring $(1,1,1)$ planes are antiparallel, and (iv) a different ferrimagnetic (FIP) phase where the Fe spins are aligned ferromagnetically if the $\mathrm{Fe}$ are in the correct positions, and antiferromagnetically if the Fe ions occupy Mo sites because of the antisite defects. In the absence of disorder, we have checked that other phases with canted spins have higher free energy. Note that the above ansätze define the average magnetization at the Fe sites, but that thermal fluctuations are also included.

\section{RESULTS}

\section{A. Phase diagram}

The phase diagram of $\mathrm{Sr}_{2-x} \mathrm{La}_{x} \mathrm{FeMoO}_{6}$, as function of $x$ and temperature, is shown in Fig. 1 for different concentrations of antisites. In the absence of defects, we find that $T_{\mathrm{C}}$ 


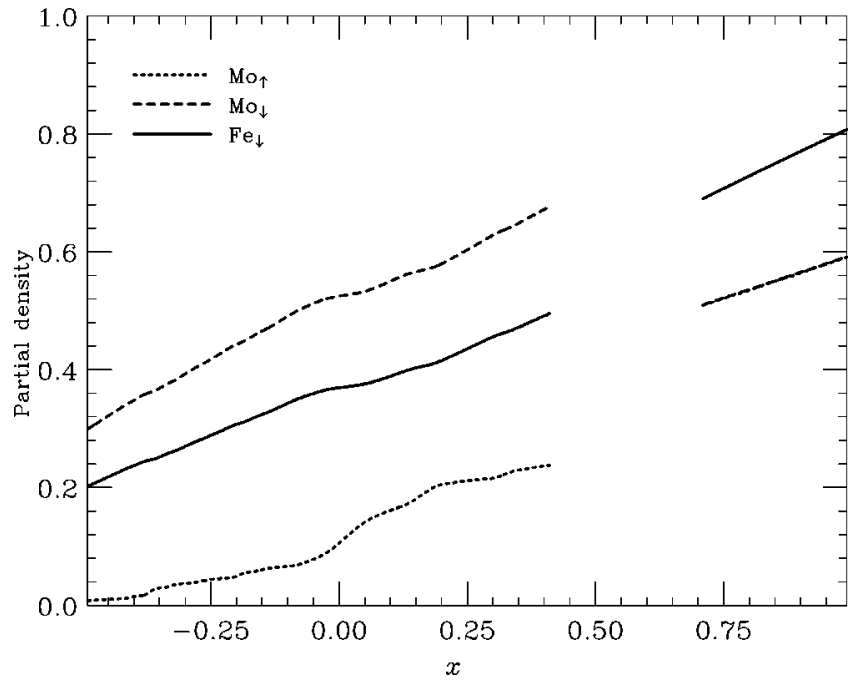

FIG. 2. Occupation of the $\mathrm{Mo}_{\uparrow}, \mathrm{Mo}_{\downarrow}$ and $\mathrm{Fe}_{\downarrow}$ as function of the doping of the conduction band. The curves give the occupancies for a $10 \%$ density of antisites defects. Note that in phases with no net magnetization, the occupancies of the $\mathrm{Mo}_{\uparrow}$ and $\mathrm{Mo}_{\downarrow}$ levels are the same.

decreases with increasing doping of the conduction band, in agreement with Ref. 22. At high, but still reasonable, dopings we find the ordered AFM phase described above. The phase transitions are first order, with regions of phase separation between them. For $x \approx 0$, the spins of the electrons at the Mo orbitals are antiparallel to the Fe core spins. We ascribe the tendency toward phases with zero magnetization, upon increasing doping, to the occupancy of the Mo orbitals which are aligned parallel to the Fe spins.

The presence of antisite defects changes significantly the phase diagram: (i) The FI phase is replaced by the FIP phase, where the spins at the Fe sites at the defects are antiparallel to the overall magnetization, (ii) the ordered AFM phase is strongly suppressed, and (iii) the value of $T_{C}$ increases as the concentration of antisites also increases ${ }^{28}$ (with our parameters choice, it reaches a maximum close to $10 \%$ ), (iv) the dependence of $T_{C}$ with the number of electrons in the conduction band is more pronounced in the presence of antisites.

These changes in the phase diagram are associated to the direct AFM interaction between spins at Fe ions which are nearest neighbors. The relevance of these interactions for the stability of a ferrimagnetic ground state was emphasized in Ref. 20. These interactions play no role in perfect materials. The antiferromagnetic interaction can be easily shown to be equivalent to a ferromagnetic one for the atoms in the $\mathrm{Fe}$ sublattice. Thus, superexchange enhances the tendency toward a ferromagnetic order in the original Fe sublattice. This effect is independent of the number of electrons in the conduction band. The saturation magnetization, on the other hand, is reduced.

\section{B. Electronic structure and saturation magnetization}

Figure 2 gives the occupancies of the different orbitals as the number of electrons in the conduction band is varied. Most of the charge is in the Mo orbitals. The variation is not

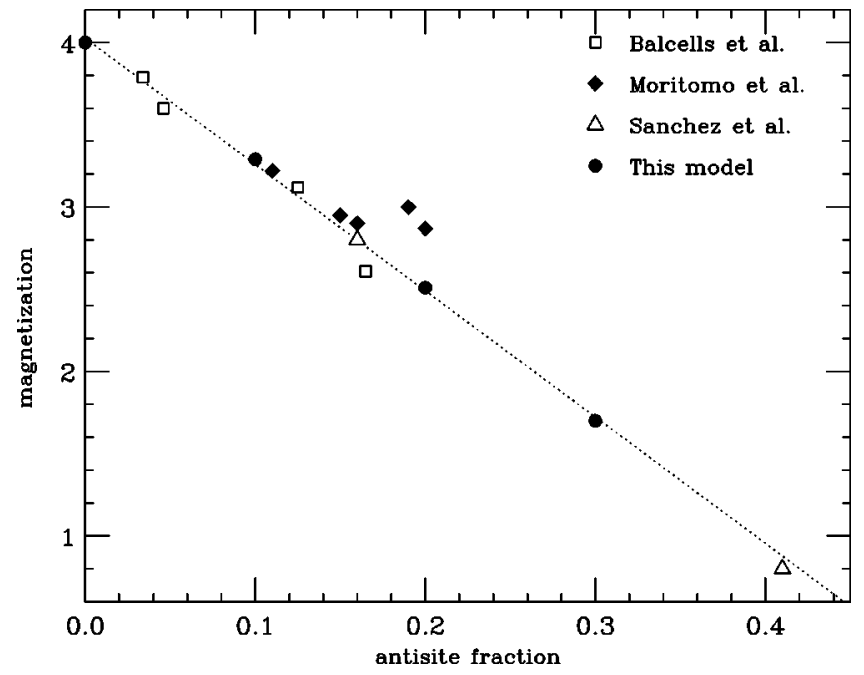

FIG. 3. Low temperature magnetization ( $\mu_{B}$ per formula unit) of $\mathrm{Sr}_{2} \mathrm{FeMoO}_{6}$ as function of the concentration of antisite defects $y$. Experimental results are from Refs. 9,11,14.

linear, indicating that a rigid band picture is not valid. ${ }^{17}$ There are sharp changes at the phase transitions.

At low temperatures, the spins at antisites tend to be antiparallel to the magnetization, as shown in Fig. 1. This implies that the saturation magnetization is reduced with respect to the ordered case. The total magnetization of the core spins and the conduction electrons, is shown in Fig. 3. The calculated magnetization is well fitted by the line $M_{\mathrm{S}}=(4.0$ $-7.7 y) \mu_{B}$, where $y$ is the antisite density. Experimental results from Refs. 9,11,14. are added for comparison. Note that the decrease in the magnetization does not lead to a lowering of the Curie temperature, as discussed above.

\section{CONCLUSIONS}

We have studied the magnetic phase diagram of the doped double perovskites, $\mathrm{Sr}_{2-x} \mathrm{La}_{x} \mathrm{FeMoO}_{6}$. We have analyzed the influence of antisite defects, on the phase diagram.

In clean systems, we find that as the number of electrons in the conduction band increases, the critical temperature decreases, in agreement with previous calculations. ${ }^{22}$ This variation is due to the increased filling of the $\mathrm{Mo}_{\uparrow}$ band, which reduces the double exchangelike mechanism which tends to align the Fe moments. At sufficiently high dopings, we find ordered phases without net magnetization, which enhance the delocalization of both the $\mathrm{Mo}_{\uparrow}$ and $\mathrm{Mo}_{\downarrow}$ bands. The transitions between these phases tend to be first order, with regions of phase separation between them. Electrostatic effects will prevent the existence of phase separation at macroscopic scales, leading to a domain structure at mesoscopic scales. $^{29}$

Antisite disorder induces significant changes in the phase diagram. The ordered ferrimagnetic phase is replaced by a different ferrimagnetic phase where the Fe spins at defects are antiparallel to the bulk magnetization (the FIP phase, see Fig. 1). Antiferromagnetism at finite dopings is suppressed. The saturation magnetization in the FIP phase is reduced, although the Curie temperature tends to increase with the 
number of Fe in Mo positions, ${ }^{30}$ due to the direct AFM exchange between $\mathrm{Fe}$ ions which are nearest neighbors. ${ }^{28}$

Note that, in order to study compounds with different number of carriers in the conduction band, the presence of vacancies and changes in the Fe-O-Mo bond angles, Fe/ Mo-O distance, and in the energy splitting $\Delta$ can influence the results. These effects need to be extracted from the available experimental data and incorporated in the model Hamiltonian (6). In addition, the reduction in the magnetization can lead to difficulties in determining the value of the Curie temperature. Our calculations give the temperature at which the magnetization vanishes, which can differ from the temperature at which the magnetization changes at a faster rate.

We have not studied transport properties, although it seems likely that the variation of the magnetic structure near defects will lead to significant changes in a half metallic system. ${ }^{17}$ We have also not analyzed other effects of the electron-electron interaction, such as the existence of a Mott transition to an insulating state, found in the related compound $\mathrm{Sr}_{2} \mathrm{FeWO}_{6} \cdot{ }^{24,20}$ We think, however, that our model includes all relevant interactions required to study the magnetic properties of the metallic state of double perovskites. Similar models provide a good understanding of the magnetic properties of the half metallic manganite oxides (such as $\left.\mathrm{La}_{1-x} \mathrm{Ca}_{x} \mathrm{MnO}_{3}\right) .{ }^{26,27}$ In summary, we find a rich phase diagram for $\mathrm{Sr}_{2-x} \mathrm{La}_{x} \mathrm{FeMoO}_{6}$, which is significantly modified in the presence of defects.

\section{ACKNOWLEDGMENTS}

We are thankful to J. Blasco, J.M.D. Coey, J.M. de Teresa, J. Fontcuberta, M. García-Hernández, M.R. Ibarra, J. L. Martínez, L. Morellón, D. D. Sarma, and D. Serrate, M. Venkatesan, and especially to V. Laliena, for helpful discussions. V.M.M. was supported by E.C. Contract No. HPMF-CT2000-00450 and by a Ramón y Cajal contract (Spain). Financial support from Grants No. PB96-0875, FPA2000-0956, FPA2000-1252, FPA2001-1813 (MCyT, Spain), and 07N/ 0045/98 (C. Madrid) are acknowledged.
${ }^{1}$ A.W. Sleight and J.F. Weiher, J. Phys. Chem. Solids 33, 679 (1972).

${ }^{2}$ K.I. Kobayashi, T. Kimura, H. Sawada, K. Terakura, and Y. Tokura, Nature (London) 395, 677 (1998); Phys. Rev. B 59, 11159 (1999).

${ }^{3}$ A.S. Ogale, S.B. Ogale, R. Ramesh, and T. Venkatesan, Appl. Phys. Lett. 75, 537 (1999).

${ }^{4}$ Y. Moritomo, Sh. Xu, T. Akimoto, A. Machida, N. Hamada, K. Ohoyama, E. Nishibori, M. Takata, and M. Sakata, Phys. Rev. B 62, 14224 (2000).

${ }^{5}$ D.D. Sarma, R. Nagarajan, S. Majumdar, A. Kumar, G. Nalini, and T.N. Guru Row, Solid State Commun. 114, 465 (2000).

${ }^{6}$ J. Gopalakrishnan, A. Chattopadhyay, S.B. Ogale, T. Venkatesan, R.L. Greene, A.J. Millis, K. Ramesha, B. Hannoyer, and G. Marest, Phys. Rev. B 62, 9538 (2000).

${ }^{7}$ C. Ritter, M.R. Ibarra, L. Morellón, J. Blasco, J. García, and J.M. De Teresa, J. Phys.: Condens. Matter 12, 8295 (2000).

${ }^{8}$ J. Navarro, C. Frontera, Ll. Balcells, B. Martínez, and J. Fontcuberta, Phys. Rev. B 64, 092411 (2001).

${ }^{9}$ L1. Balcells, J. Navarro, M. Bibes, A. Roig, B. Martínez, and J. Fontcuberta, Appl. Phys. Lett. 78, 781 (2001).

${ }^{10}$ M. García-Hernández, J.L. Martínez, M.J. Martínez-Lope, M.T. Casais, and J.A. Alonso, Phys. Rev. Lett. 86, 2443 (2001).

${ }^{11}$ Y. Moritomo, N. Shimamoto, Sh. Xu, A. Machida, E. Nishibori, M. Takata, M. Sakata, and A. Nakamura, Jpn. J. Appl. Phys. 40, L672 (2001).

${ }^{12}$ J.M. Greneche, M. Venkatesan, R. Suryanarayanan, and J.M.D. Coey, Phys. Rev. B 63, 174403 (2001).

${ }^{13}$ D. Serrate, J.M. De Teresa, J. Blasco, M.R. Ibarra, and L. Morellón, Appl. Phys. Lett. 80, 4573 (2002).

${ }^{14}$ D. Sánchez, J.A. Alonso, M. García-Hernández, M.J. MartínezLope, J.L. Martínez, and A. Mellergard, Phys. Rev. B 65, 104426 (2002).

${ }^{15}$ M. Tovar, M.T. Causa, A. Butera, J. Navarro, B. Martínez, J. Fontcuberta, and M.C.G. Passeggi, Phys. Rev. B 66, 024409 (2002).
${ }^{16}$ M. Venkatesan, M. Grafoute, A.P. Douvalis, J.-M. Greneche, R. Suryanarayanan, and J.M.D. Coey, J. Magn. Magn. Mater. 242245, 744 (2002).

${ }^{17}$ T. Saha-Dasgupta and D.D. Sarma Phys. Rev. B 64, 064408 (2001).

${ }^{18}$ T.M. Rearick, G.L. Catchen, and J.M. Adams, Phys. Rev. B 48, 224 (1993).

${ }^{19}$ D.D. Sarma, P. Mahadevan, T. Saha-Dasgupta, S. Ray, and A. Kumar, Phys. Rev. Lett. 85, 2549 (2000).

${ }^{20}$ I.V. Solovyev, Phys. Rev. B 65, 144446 (2002).

${ }^{21}$ S. Ray, P. Mahadevan, A. Kumar, D.D. Sarma, R. Cimino, M. Pedio, L. Ferrari, and A. Pesci, Phys. Rev. B 67, 085109 (2003).

${ }^{22}$ A. Chattopadhyay and A.J. Millis, Phys. Rev. B 64, 024424 (2001).

${ }^{23}$ A.A. Aligia, P. Petrone, J.O. Sofo, and B. Alascio, Phys. Rev. B 64, 092414 (2001).

${ }^{24}$ P. Petrone and A.A. Aligia, Phys. Rev. B 66, 104418 (2002).

${ }^{25}$ R. Gautier, O.K. Andersen, P. Gourgeon, J.-F. Halet, E. Canadell, and J.D. Martin (unpublished).

${ }^{26}$ J.L. Alonso, L.A. Fernández, F. Guinea, V. Laliena, and V. Martínez-Mayor, Phys. Rev. B 63, 054411 (2001); 63, 064416 (2001).

${ }^{27}$ J.L. Alonso, L.A. Fernández, F. Guinea, V. Laliena, and V. Martínez-Mayor, Nucl. Phys. B596, 587 (2001); J.L. Alonso, J.A. Capitán, L.A. Fernández, F. Guinea, and V. MartínezMayor, Phys. Rev. B 64, 054408 (2001).

${ }^{28}$ Our estimate of $J_{\mathrm{Fe}-\mathrm{Fe}} / t_{\mathrm{Fe}-\mathrm{Mo}}=0.1$ gives a lower bound to this anomalous dependence of $T_{C}$ on the number of defects. A lower, but physically reasonable, value for $t_{\mathrm{Fe}-\mathrm{Mo}}$ than the one used here, $t_{\mathrm{Fe}-\mathrm{Mo}} \approx 0.35 \mathrm{eV}$, leads to a more pronounced increase of $T_{C}$ as function of the number of antisite defects, for fixed doping.

${ }^{29}$ F. Guinea, G. Gómez-Santos, and D.P. Arovas, Phys. Rev. B 62, 391 (2000).

${ }^{30}$ M. G. Hernández (private communication). 